Bioelectrochemistry and Bioenergetics, 16 (1986) 45-54

A section of $J$. Electroanal. Chem., and constituting Vol. 212 (1986)

Elsevier Sequoia S.A., Lausanne - Printed in The Netherlands

\title{
871 - DIELECTRIC SPECTROSCOPY OF ENERGY COUPLING MEMBRANES: CHLOROPLAST THYLAKOIDS *
}

\author{
MARC SYMONS and RAFI KORENSTEIN \\ Department of Membrane Research, Weizmann Institute of Science, Rehovot 76100 (Israel) \\ CHRISTINE M. HARRIS and DOUGLAS B. KELL * $\star$ \\ Department of Botany and Microbiology, University College of Wales, Aberystwyth, Dyfed SY23 $3 D A$ \\ (Great Britain)
}

(Manuscript received June 29th 1985)

\section{SUMMARY}

(1) The dielectric properties (complex permittivity) of suspensions of chloroplast thylakoids have bcen detcrmined in the range $10 \mathrm{~Hz}$ to $13 \mathrm{MHz}$. In common with other charged membrane vesicles, thylakoids exhibit two broad dielectric dispersions corresponding to the classical $\alpha$ - and $\beta$-dispersions.

(2) Heat treatment of thylakoids, to produce blebs of a greater average radius, increases, as expected, the magnitude of the $\beta$-dispersion, but not that of the $\alpha$-dispersion. This result suggests that the $\alpha$-dispersion is not caused solely by the unrestricted tangential relaxation of the ions of the diffuse double layer.

(3) The magnitude of the $\alpha$-dispersion is strongly pH-dependent, and is negligible at the $\mathrm{pI}$ ( $\mathrm{ca}$. $\mathrm{pH}$ 4.3-4.4) of thylakoids. The $\beta$-dispersion is much less sensitive to $\mathrm{pH}$. Measurements of the magnitude of the $\alpha$ dispersion may therefore be used to obtained the pI of charged membrane vesicles.

(4) Both $\alpha$ - and $\beta$-dispersions are significantly decreased by treatment of the thylakoids with the cross-linking reagent glutaraldehyde, suggesting that the (lateral) motions (in particular) of charged membrane components contribute to the dielectric properties in this frequency range. The relaxation times observed, however, are not consistent with the view that such motions are restricted by hydrodynamic forces alone. The breadth of the dispersion remains very large, however, suggesting also a variable restriction on the genuinely tangential motions of double ions.

Science is Spectrum Analysis, Art is Photosynthesis

K. Kraus

\footnotetext{
* Contribution presented at the VIIIth International Symposium on Bioelectrochemistry and Bioenergetics, Bologna, June 24th-29th 1985.

$\star \star$ To whom correspondence should be addressed.
} 


\section{INTRODUCTION}

An understanding of the passive electrical properties of energy coupling membranes (i.e. of the inner mitochondrial, chloroplast thylakoid and bacterial cytoplasmic membranes) is assuming increasing significance, not least because of the realisation of the importance of electrical forces in electron transport-linked phosphorylation [1-5]. To this end, we have constructed a rapid, automated, frequencydomain dielectric spectrometer, capable of operating in the range $5 \mathrm{~Hz}-13 \mathrm{MHz}$ and based on commercially available apparatus [6], and have applied it to the study of the electrical organisation of a number of microbial cells, protoplasts and membrane vesicle preparations [7-11].

Classically [e.g. 12-21], it is considered that, when measurements are made with extracellular electrodes, charged biomembrane vesicles exhibit two major dielectric dispersions in this frequency range, known as the $\alpha$-and $\beta$-dispersions. The former, more slowly relaxing, dispersion is ascribed to the relaxation, tangential to the charged membrane surface, of the loosely-bound ions constituting the diffuse double layer [22-25]; it thus depends on the possession by the suspended phase of a net surface charge, and its relaxation time scales as the inverse square of the particle radius $[26,27]$. The latter, $\beta$-dispersion is ascribed to the progressive short-circuiting of the capacitance of the poorly-ion-permeable membrane, via a Maxwell-Wagner type of mechanism (e.g. Refs. 10-12 and 28). Its magnitude is therefore (for a given volume fraction) a direct function of the particle's radius and the membrane capacitance, whilsts its relaxation time is additionally related to the internal and external resistivities of the aqueous phases that the membrane serves to separate (e.g. Refs. 10-12, 19 and 28-30). Thus these mechanisms assume:

(a) a wholly unrestricted flow of double-layer ions tangential to the charged membrane surface, and

(b) that the possible lateral and rotational mobilities of membrane lipids and proteins do not contribute to the dielectric properties in this frequency range.

Our own observations to date [7-11] indicate that these assumptions are unlikely to prove correct, at least in microbial energy coupling membranes; the breadth of the dispersions observed, and the effects of chemical crosslinking reagents, suggest both:

(a) an impairment to genuinely tangential ion mobility by protein complexes raised above the surface delineated by the phospholipid head-groups, and

(b) that the lateral mobility (in particular) of membrane proteins and lipids can contribute significantly to the dielectric properties of energy-coupling biomembrane vesicles.

The observed relaxation time(s) of the latter type of mechanism [7,9-11,31] are not obviously consistent with the view that the long-range mobility of membrane components is restricted by hydrodynamic forces alone, a point that is of special significance in view of present uncertainty concerning the randomness of the organisation of the protein complexes in the bacterial cytoplasmic membrane $[32,33]$. 
Chloroplast thylakoids have certain advantages over respiratory bacteria for the study of bioelectrical phenomena in energy coupling membranes, including their possession of pigments with field-sensitive spectral properties (515 nm shift) [34-36] and the ability to stimulate delayed [37-39] and thermo-luminescence [40,41] and vesicle rotation [29] by appropriate external electric fields. Against this, their size heterogeneity and reversible ionic-strength-dependent organisation into granal stacks (e.g. Ref. 42) can make the interpretation of data somewhat complicated, although this problem may to some extent be alleviated by the production of spherical blebs by hypotonic or thermal means [37-39,43]. The radius of chloroplasts is, of course, somewhat greater than those of the microbial systems that we have thus far studied. It was therefore of interest, in view of the foregoing, to carry out a study of the frequency-dependent dielectric properties of chloroplast thylakoids, an investigation which forms the subject of the present article.

\section{EXPERIMENTAL}

\section{Preparation of thylakoids}

Class $\mathrm{C}$ chloroplasts from lettuce were prepared as previously described [43]. The chloroplasts were washed and stored in a medium containing $0.4 M$ sucrose and 3 $\mathrm{m} M$ Tris (pH 7.5). Storage of chloroplasts, supplemented by $30 \%$ ethylene glycol, was at $-20^{\circ} \mathrm{C}$. Before use, they were thawed, washed once in $0.4 \mathrm{M}$ sucrose and resuspended in the same to a chlorophyll concentration of $3.8 \mathrm{mg} / \mathrm{cm}^{3}$. Thylakoid suspensions were then treated in one of the following ways.

\section{Treatment with the cross-linking agent glutaraldehyde}

A thylakoid suspension prepared as above was titrated to $\mathrm{pH} 7.2$ with $\mathrm{KOH}$ and incubated at $20^{\circ} \mathrm{C}$ for $1 \mathrm{hr}$ in the presence of $5 \%(w / V)$ glutaraldehyde. A further sample at the identical $\mathrm{pH}$ was not so treated, but its conductivity, as measured at $10 \mathrm{~Hz}$, was adjusted with $0.1 M \mathrm{KCl}$ so that it was the same as that of the glutaraldehyde-treated sample.

\section{Heat treatment}

Thylakoid samples ( $\mathrm{pH} 7.2)$ were incubated at $50^{\circ} \mathrm{C}$ for $1 \mathrm{hr}$, and then allowed to cool at $20^{\circ} \mathrm{C}$. The conductance (at $10 \mathrm{~Hz}$ ) of a control sample was then titrated to that of the heat-treated thylakoids (after cooling to $20^{\circ} \mathrm{C}$ ) using $0.1 \mathrm{M} \mathrm{KCl}$. The heat treatment produced spherical blebs in approximately $80 \%$ of the chloroplasts. These blebs appeared to be similar to those obtained by hypotonic treatment [43] with the differences that they were more homogeneous in size and smaller, with an average radius of about $2 \mu \mathrm{m}$. Furthermore, they retain most of the original chloroplast which appears as a large patch or conglomerate which is attached to the bleb. A detailed analysis of the effects of heat treatment on the structure and function of chloroplasts will be presented elsewhere. The reason for choosing heat treatment over hypotonic treatment is that with the former we can obtain unilamel- 
lar bleb formation in suspensions concentrated enough to give a reasonable dielectric increment.

\section{Adjustment of $p H$}

The sample of thylakoids was diluted to a chlorophyll concentration of 1.75 $\mathrm{mg} / \mathrm{cm}^{3}$ with $0.4 M$ sucrose, to lower the conductivity yet further, and then titrated using small volumes of $1 \mathrm{M} \mathrm{HCl}$ to $\mathrm{pH}$ values of $6.9,5.5,4.6$ and 4.4. In each case the conductivity at $10 \mathrm{~Hz}$ was adjusted with $0.1 \mathrm{M} \mathrm{KCl}$ such that it was comparable to the (otherwise most conducting) sample at $\mathrm{pH}$ 4.4. The adjustment of conductivity is extremely important in view of the possible significance of electrode polarisation to measured dielectric spectra at low frequencies in conductive media $[14,44-46]$.

\section{Dielectric spectroscopy}

The dielectric spectrometer has been described in detail elsewhere $[6,46]$. The system consists of a Hewlett-Packard 4192A Impedance Analyser driven, via an interface conforming to the IEEE-488 (1978) standard, by an HP85A microcomputer and connected to a HP 9121D disc storage device and an HP 7225A plotter. Programs were written in BASIC. Similar instrumentation has been described by others $[20,47]$. The electrode cell was contained within a thermostatted water jacket, and all measurements were carried out at $20^{\circ} \mathrm{C}$. The $\mathrm{Pt}$ electrodes, which led directly to the impedance analyser, were of the pin-type [48] and were given a heavy coat of Pt black. The dimensions of the electrodes were $1 \mathrm{~mm} \times 8 \mathrm{~mm}$ and their separation ca. $8 \mathrm{~mm}$.

\section{RESULTS AND DISCUSSION}

Figure 1 shows dielectric spectra in the range $10 \mathrm{~Hz}$ to $13 \mathrm{MHz}$ of:

(i) a suspension of broken chloroplasts, and

(ii) of heat-treated chloroplasts at the same chlorophyll concentration, prepared as described in the experimental section. Dielectric increments were strictly dependent upon the concentration of the suspended phase. As far as we are aware, these are the first dielectric spectra of chloroplast thylakoids to be made in this range, although measurements of d.c. photoconductivity $[49,50]$ and time-dependent photoelectrical signals [51] have been reported, and Gordon [52-54] and Göttman and Dittrich [55] reported on the dielectric properties of dark and illuminated chloroplasts at higher frequencies. None of these studies is directly comparable with the present observations. Figure 1 also shows the (apparent) permittivity and conductivity of an ionic solution of the same (low-frequency) conductivity, by which one may discern the extent of the electrode polarisation at the lower frequencies. It is evident that both suspensions exhibit two broad dispersions (i.e. frequency-dependent dielectric properties) down to, but not below, frequencies in the decade $10 \mathrm{~Hz}$ to 100 $\mathrm{Hz}$. The heat treatment clearly serves to increase the magnitude of the more rapidly relaxing ( $\beta-)$ dispersion, consistent with the view that this dispersion is dominated 


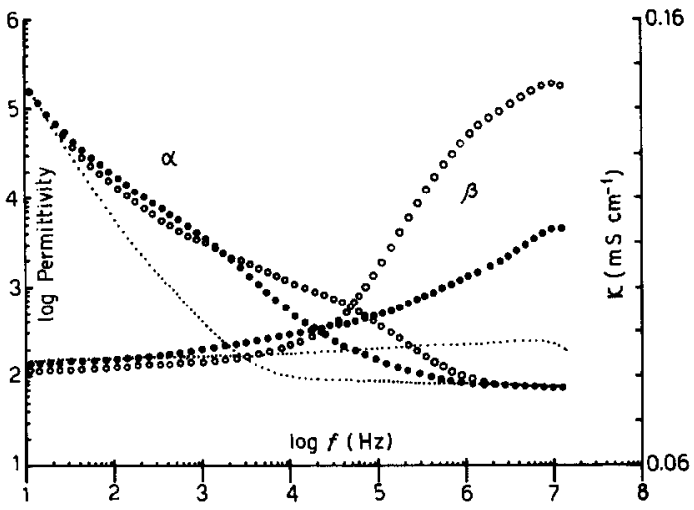

Fig. 1. Dielectric spectra of broken chloroplasts. Thylakoids $(\bullet)$ and blebs $(O)$ were prepared and suspended as described in the experimental section. The dielectric spectrum of a $\mathrm{KCl}$ solution of the same conductivity is also shown $(\cdots \cdots)$. The conductivity increases and the permittivity decreases as the frequency increases. The $\alpha$ - and $\beta$-dispersions are indicated. $\mathrm{pH}=7.2, T=20^{\circ} \mathrm{C}$, thylakoid concentration $=3.8 \mathrm{mg} / \mathrm{cm}^{3}$ chlorophyll.

by a Maxwell-Wagner type of mechanism, whose dielectric increment is given (for spherical vesicles suspended at a volume fraction less than 0.2) by $9 \operatorname{PrC}_{m} / 4 \epsilon_{0}$, where $P$ is the volume fraction of the suspended phase, $r$ the (spherical) radius, $C_{m}$ the membrane capacitance per unit area and $\epsilon_{0}$ the permittivity of free space $\left(=8.854 \times 10^{-14} \mathrm{~F} / \mathrm{cm}\right)(e . g$ Refs. $6,10-12$ and 28$)$, so that the magnitude of this dispersion is a linear function of the vesicle radius. The small but discernible apparent decrease in conductivity at the very highest frequencies, both of the solutions and suspensions studied, is an artefact of the measuring system [8]. It would be of great interest to be able to derive a value for $C_{m}$ from measurements such as those in Fig. 1 by means of the formula given above. However, we resist the temptation to do this, since

(a) the radii of the material, and their distribution, are not known with exact precision,

(b) there is no treatment available for suspended membrane vesicles with a fractal shape such as those of thylakoids, and

(c) there are reasons to believe (see later) that the Maxwell-Wagner mechanism is not the sole mechanism underlying the $\beta$-dispersion.

Classically, the $\alpha$-dispersion in (charged) membrane vesicles is thought to be ascribable to the relaxation, tangential to the membrane surface, of the ions of the double layer [7,22-25]. However, according to such a mechanism, the increase in thylakoid radius caused by the blebbing treatment, and clearly discernible in the frequency range ascribed to the $\beta$-dispersion, should cause a related increase both in magnitude and relaxation time of the $\alpha$-dispersion, an expectation which was not realised (Fig. 1).

The possession of an $\alpha$-dispersion generally, in colloidal and membrane vesicle suspensions, is thought to be dependent upon the possession by the suspended 


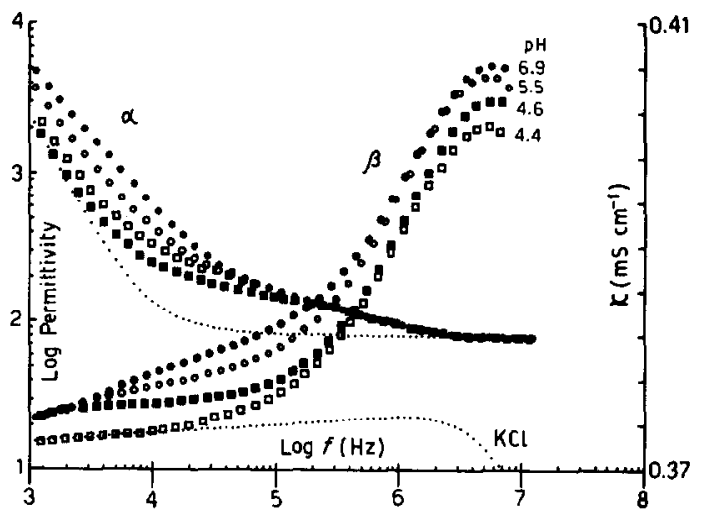

Fig. 2. Effect of $\mathrm{pH}$ on the dielectric properties of thylakoids. Thylakoids $\left(1.75 \mathrm{mg}\right.$ chlorophyll $\left./ \mathrm{cm}^{3}\right)$ were prepared, and their conductivity and $\mathrm{pH}$ adjusted, as described in the experimental section. $\mathrm{pH}$ values were $4.4(\square), 4.6(\square), 5.5(O)$ and $6.9(\bullet)$.

phase of a net surface charge. We therefore studied the influence of pH upon the dielectric properties of isolated thylakoids, since it is known that this surface charge (density) is strongly pH-dependent, with a pI of approximately 4.3 [56-58]. The data obtained are shown in Fig. 2, where it may be observed that the possession of a significant $\alpha$-dispersion is strictly dependent upon the possession by the thylakoids of a net surface charge, since it is obviated at the pI of the thylakoids. The magnitude of the $\beta$-dispersion is only very slightly changed by the $\mathrm{pH}$, the small extent being masked by the scale used in the figure. Whilst it would have been desirable to titrate the $\mathrm{pH}$ to values more acid than that of the thylakoid $\mathrm{pI}$, so as to see whether or not an $\alpha$-dispersion was again seen, we were unfortunately unable to test this due to the excessive conductivity induced by the large quantities of acid that had to be added in such a case.

Thus, a seeming paradox exists, in that the pH dependence of the possession of an $\alpha$-dispersion clearly demonstrated that a net surface charge is required (Fig. 2), whilst the radius-dependence of the magnitude and relaxation time of the $\alpha$-dispersion are not obviously consistent with the view that the $\alpha$-dispersion is caused solely by the unhindered relaxation of double layer ions. Our own studies with bacterial chromatophores, protoplasts and membrane vesicles [7-11], and those of Uhlendorf [59] with liposomes, have drawn attention to the possibility that the diffusion of charged membrane lipids and proteins may contribute to the passive electrical properties of this type of system, and this type of mechanism would also be contingent upon the possession by the membranes of a net surface charge, a prediction that was borne out in bacterial chromatophores [8]. We therefore studied the effect of the cross-linking reagent glutaraldehyde on the dielectric properties of the thylakoid suspension (Fig. 3). The reagent served significantly to decrease the magnitude of both the $\alpha$ - and $\beta$-dispersions, although neither was abolished. The chemistry of the glutaraldehyde-membrane interaction is not uncomplicated, but it should be noted that glutaraldehyde is not in general thought to alter the surface 


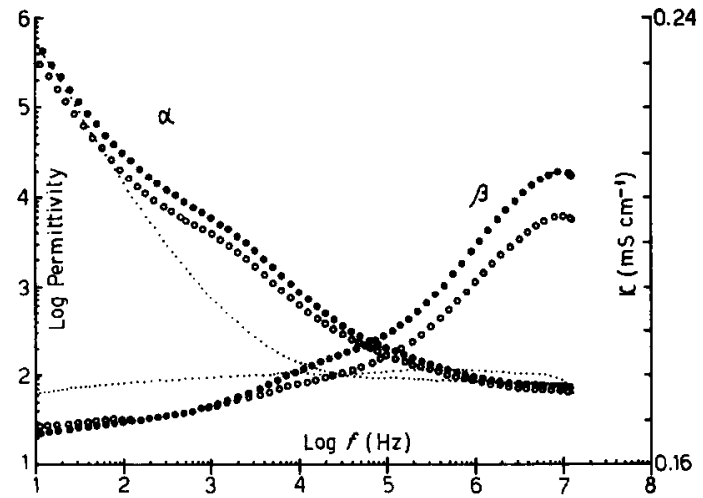

Fig. 3. Effect of glutaraldehyde on the dielectric properties of thylakoids. Thylakoids were either suspended as isolated ( $\bullet$ or were treated with glutaraldehyde as described in the experimental section (O). The apparent dielectric properties of an ionic solution of comparable conductivity are also displayed $(\cdots)$

charge density in such systems $[69,61]$. However, treatment of Ascites cells with formaldehyde [62] and of maize root protoplasts with glutaraldehyde [63], actually caused an increase in the density of surface negative charges, as judged by measurements of electrophoretic mobility (zeta potential). In broken chloroplasts, electrophoretic mobility measurements [58] did indicate that glutaraldehyde could decrease the surface charge density, whilst measurements using 9-amino acridine fluorescence [64] suggested that the reagent increased the surface charge density. Since the latter paper [64] showed that the binding acridine molecules gave higher estimates of surface charge density than did other screening cations, it is to be assumed that the complex interfacial structure of the thylakoid membrane is not adequately modelled by the simplest type of Gouy-Chapman theory alone [58,64-66], and we note also that, at low ionic strengths, the apparent zeta potential is not independent of the surface conductivity of the suspended phase $[42,66,67]$. The balance of evidence therefore does not lead us to suppose that the effects of glutaraldehyde might alone be mediated by any possible decrease in surface charge density, particularly since glutaraldehyde is thought to react predominantly with positively charged amino groups. If the cross-linking reagent was serving to inhibit the lateral motions of membrane components, that were freely diffusible in the membrane, the relaxation time $\left[\tau=(1 / 2) \pi f_{c}\right.$, where $f_{c}$ is the characteristic frequency] would be given by $\tau=r^{2} / 2 D$, where $r$ is the (spherical) radius, neglecting invaginations and stacking, and $D$ the lateral diffusion coefficient $[19,68-70]$. Thus $f_{c}=$ $D / \pi r^{2}$, so that even if $D$ values were as great as $10^{-8} \mathrm{~cm}^{2} \mathrm{~s}^{-1}$, characteristic of phospholipids whose diffusion is restricted by hydrodynamic forces alone [71,72], and the thylakoid radius was as little as $0.25 \mu \mathrm{m}$, the $f_{c}$ values would still lie below $10 \mathrm{~Hz}$. Thus the glutaraldehyde-sensitive motions cannot be explained by invoking a free and random lateral diffusibility of membrane components throughout the membrane phase. Of course the real two-dimensional distance moved by a thylakoid 


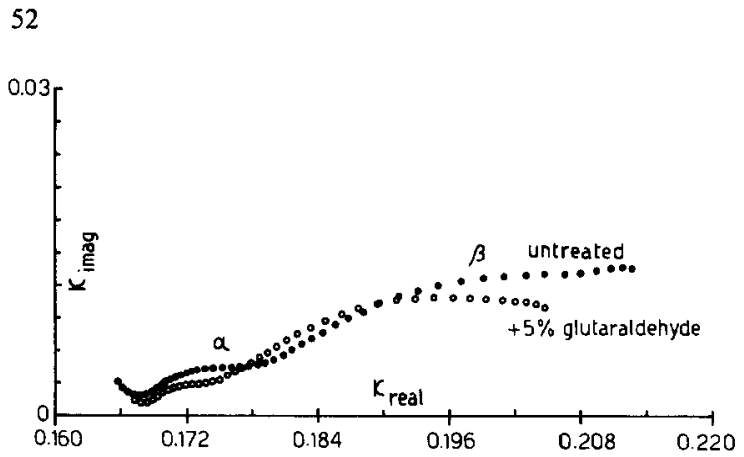

Fig. 4. Effect of glutaraldehyde on the dielectric properties of thylakoids. The figure shows a complex conductivity plot [6] of the data of Fig. 3. (0) Untreated thylakoids; (O) thylakoids treated with $5 \%$ glutaraldehyde. $\kappa_{\text {real }}=$ real part of the complex conductivity, $\kappa_{\text {imag }}=$ imaginary part of the complex conductivity.

protein would be much greater, and its relaxation time much longer, than that calculated herein; however, the purpose of the calculation was to indicate that some restriction on membrane component mobility is an important feature of thylakoid membrane organisation. This conclusion concords with that obtained in previous dielectric work in microorganisms [7,9-11].

The complex conductivity plots of the data of Fig. 3 are given in Fig. 4, which serves not only more easily to show the diminution of both $\alpha$-and $\beta$-dispersions by the glutaraldehyde treatment but also to indicate the very substantial breadth of the dispersions, a breadth much greater than can be accounted for in terms of a distribution of vesicle radii within the confines of the classical explanations of the $\alpha$ - and $\beta$-dispersions [11]. Possible explanations of the observations include the ideas that:

(1) there is a strong coupling between double-layer and membrane component motions $[9-11,66,73,74]$, and

(2) that neither of these types of motion are restricted by hydrodynamic forces alone.

The restrictions on the tangential motions of double layer ions may arise additionally not only from the overlap of double layers in some cases [42] but from the molecular roughness of the thylakoid surface occasioned by the fact that the protein complexes are raised above the membrane surface [9-11]. Finally, Schiffrin [75] has pointed out an explanation based upon the Marangoni effect [76,77] that could prove of value in accounting for some of the dielectric properties of charged membrane vesicles. Further studies with proteases and lipases might shed further light on these mechanisms.

\section{ACKNOWLEDGEMENT}

D.B.K. and C.M.H. thank the Science and Engineering Research Council, U.K. and ICI Agricultural Division for financial support. We are grateful to Sian Evans for typing the manuscript. 


\section{REFERENCES}

1 P. Mitchell, Science, 206 (1979) 1148.

2 S.J. Ferguson and M.C. Sorgato, Annu. Rev. Biochem., 51 (1982) 185.

3 D.G. Nicholls, Bioenergetics, Academic Press, New York, 1982.

4 H.V. Westerhoff, B.A. Melandri, G. Venturoli, G.F. Azzone and D.B. Kell, Biochim. Biophys. Acta, 768 (1984) 257.

5 S.J. Ferguson, Biochim. Biophys. Acta, 811 (1985) 47.

6 C.M. Harris and D.B. Kell, Bioelectrochem. Bioenerg., 11 (1983) 15.

7 D.B. Kell, Bioelectrochem. Bioenerg., 11 (1983) 415.

8 C.M. Harris, G.D. Hitchens and D.B. Kell in Charge and Field Effects in Biosystems, M.J. Allen and P.N.R. Usherwood (Editors), Abacus Press, Tunbridge Wells, 1984, p. 179.

9 C.M. Harris and D.B. Kell, Eur. Biophys. J., 12 (1985) 11.

10 D.B. Kell and C.M. Harris, Eur. Biophys. J., 12 (1985) 181.

11 D.B. Kell and C.M. Harris, J. Bioelectricity, 4 (1985) 317.

12 H.P. Schwan in Advances in Biological and Medical Physics, J.H. Lawrence and C.A. Tobias (Editors), Academic Press, New York, 1957, Vol. 5, p. 147.

13 E.L. Carstensen and R.E. Marquis in Spores VI, P. Gerhardt, R.N. Costilow and H.L. Sadoff (Editors), American Society for Microbiology, Washington, DC, 1975, p. 563.

14 E.H. Grant, R.J. Sheppard and G.P. South, Dielectric Behaviour of Biological Molecules in Solution, Oxford University Press, London, 1978.

15 O.F. Schanne and E.R.P. Ceretti, Impedance Measurements in Biological Cells, Wiley, Chichester, 1978.

16 R. Pethig, Dielectric and Electronic Properties of Biological Materials, Wiley, Chichester, 1979.

17 W.R. Adey, Physiol. Rev., 61 (1981) 435.

18 H.P. Schwan, A.C.S. Symp. Ser., 157 (1981) 109.

19 U. Zimmermann, Biochim. Biophys. Acta, 694 (1982) 227.

$20 \mathrm{~K}$. Asami and A. Irimajiri, Biochim. Biophys. Acta, 769 (1984) 370.

21 R. Pethig, IEEE Trans. Electrical Insulation, EI-19 (1984) 453.

22 G. Schwarz, J. Phys. Chem., 66 (1962) 2636.

23 H.P. Schwan, G. Schwarz, J. Maczuk and H. Pauly, J. Phys. Chem., 66 (1962) 2626.

24 C.W. Einolf and E.L. Carstensen, J. Phys. Chem., 75 (1971) 1091.

25 S.S. Dukhin and V.N. Shilov, Dielectric Phenomena and the Double Layer in Disperse Systems and Polyelectrolytes, Wiley, Chichester, 1974.

26 H.P. Schwan, S. Takashima, V.K. Miyamoto and W. Stoeckenius, Biophys. J., 10 (1970) 1102.

27 W.R. Redwood, S. Takashima, H.P. Schwan and T.L. Thompson, Biochim. Biophys. Acta, 255 (1972) 557.

28 H.P. Schwan and K.R. Foster, Proc. IEEE, 68 (1980) 104.

29 W.M. Arnold, B. Wendt, U. Zimmermann and R. Korenstein, Biochim. Biophys. Acta, 813 (1984) 117.

30 D.B. Kell and A. Burns. J. Bioelectricity. 5 (1986) 139.

31 D.B. Kell, EBEC Rep., 3 (1984) 645.

32 D.B. Kell, Trends Biochem. Sci., 9 (1984) 86.

33 D.B. Kell and H.V. Westerhoff in Organised Multienzyme Systems: Catalytic Properties, G.R. Welch (Editor), Academic Press, New York, 1985, p. 63.

34 P. Gräber, Curr. Top. Membr. Trans., 16 (1981) 215.

35 E. Schlodder, P. Gräber and H.T. Witt in Electron Transport and Photophosphorylation, J. Barber (Editor), Elsevier, Amsterdam, 1982, p. 105.

36 W. Junge and J.B. Jackson in Photosynthesis: Energy Conversion by Plants and Bacteria, Govindjee (Editor), Academic Press, New York, 1982, p. 589.

37 B.G. De Grooth and H.J. van Gorkom, Biochim. Biophys. Acta, 635 (1981) 445.

38 D.L. Farkas, R. Korenstein and S. Malkin, Biophys. J., 45 (1984) 363.

39 D.L. Farkas, S. Malkin and R. Korenstein, Biochim. Biophys. Acta, 767 (1984) 507. 
40 P.P. Knox and G. Garab, Photochem. Photobiol., 35 (1982) 733.

41 P.P. Knox, P.S. Venediktov,. A.A. Kononenko, G.I. Garab and A. Faludi-Daniel, Photochem. Photobiol., 40 (1984) 119.

42 J. Barber, Biochim. Biophys. Acta, 594 (1980) 253.

43 M. Symons, S. Malkin and R. Korenstein, Biochim. Biophys. Acta, 767 (1984) 223.

44 H.P. Schwan in Physical Techniques in Biological Research, W.L. Nastuk (Editor), Academic Press, New York, 1963, Vol. VIB, p. 323.

45 H.P. Schwan, Biophysik, 3 (1966) 181.

46 D.B. Kell in Biosensors: Fundamentals and Applications, A.P.F. Turner and I. Karube (Editors), Oxford University Press, Oxford, 1985, p. 429.

47 J.B. Kosterich, K.R. Foster and S.R. Pollack, IEEE Trans. Biomed. Eng., BME-30 (1983) 81.

48 H. Pauly and H.P. Schwan, Biophys. J., 6 (1966) 621.

49 K.J. McCree, Biochim. Biophys. Acta, 102 (1965) 96.

50 F.F. Litvin and V.I. Zvalinskii, Biophysics, 13 (1968) 286.

51 H.W. Trissl, U. Kunze and W. Junge, Biochim. Biophys. Acta, 682 (1982) 364.

52 W. Gordon, J. Membr. Biol., 10 (1972) 193.

53 W. Gordon, J. Membr. Biol., 8 (1972) 97.

54 W. Gordon, J. Membr. Biol., 12 (1973) 385.

55 O. Göttman and A. Dittrich, J. Phys. E. Sci. Instr., 17 (1985) 772

56 H.Y. Nakatani, J. Barber and J.A. Forrester, Biochim. Biophys. Acta, 504 (1978) 215.

57 H.Y. Nakatani and J. Barber, Biochim. Biophys. Acta, 591 (1980) 82.

58 A.H.C.M. Schapendonk, A.M. Hemrika-Wagner, A.P.R. Theuvenet, H.W. Wong Fong Sang, W.J. Vredenberg and R. Kraayenhof, Biochemistry, 19 (1980) 1922.

59 V. Uhlendorf, Biophys. Chem., 20 (1984) 261.

60 G.E. Means and R.E. Feeney, Chemical Modification of Proteins, Holden-Day, San Francisco, 1971, p. 89.

61 K. Peters and F.M. Richards, Annu. Rev. Biochem., 46 (1977) 523.

62 C. Roeder, G.M.W. Cook, G. Haemmerli and P. Strauli, J. Cell. Sci., 12 (1973) 235.

63 A. Senn and P.E. Pilet, Z. Pflanzenphysiol., 102 (1980) 19.

64 W.S. Chow and J. Barber, J. Biochem. Biophys. Meth., 3 (1980) 175.

65 D.B. Kell, Biochim. Biophys. Acta, 549 (1979) 55.

66 J.M.G. Torres-Pereira, H.W. Wong Fong Sang, A.P.R. Theuvenet and R. Kraayenhof, Biochim. Biophys. Acta, 767295.

67 C.W. Einolf and E.L. Carstensen, Biochim. Biophys. Acta, 148 (1967) 506.

68 H.W. Huang, J. Theoret. Biol., 40 (1973) 11.

69 A.E. Sowers and C.R. Hackenbrock, Proc. Natl. Acad. Sci. USA, 78 (1981) 6246.

70 D.B. Kell, Trends Biochem. Sci., 9 (1984) 86.

71 R. Peters, Cell Biol. Int. Rep., 5 (1981) 733.

72 W.L.C. Vaz, Z.I. Derzko and K.A. Jacobson in Membrane Reconstitution, G. Poste and G.L. Nicolson (Editors), Elsevier, Amsterdam, 1982, p. 83.

73 S. McLaughlin and M-m. Poo, Biophys. J., 34 (1981) 85.

74 J.R. Rabinowitz, J. Theoret. Biol., 99 (1982) 377.

75 D. Schiffrin, personal communication, 1985.

76 T.S. Sørenson, J. Chem. Soc. Faraday II, 76 (1980) 1170.

77 H.H.J. Girault and D.J. Schiffrin, Biochim. Biophys. Acta, submitted. 